\title{
Erratum zu: Quantencomputing kompakt
}

\section{Erratum zu: B. Just, Quantencomputing kompakt, IT kompakt, https://doi.org/10.1007/978-3-662-61889-9}

Die Originalversion des Buches wurde mit falschen Typos und Gleichungen veröffentlicht. Dies wurde in den folgenden Punkten korrigiert.

\section{Titelei:}

Auf S. VII, letzter Absatz: „zu schaffen“ wurde korrigiert zu „schaffen“"

Die aktualisierte Version dieses Buches finden Sie unter

https://doi.org/10.1007/978-3-662-61889-9

https://doi.org/10.1007/978-3-662-61889-9_1

https://doi.org/10.1007/978-3-662-61889-9_2

https://doi.org/10.1007/978-3-662-61889-9_3

https://doi.org/10.1007/978-3-662-61889-9_4

https://doi.org/10.1007/978-3-662-61889-9_5

https://doi.org/10.1007/978-3-662-61889-9_8

https://doi.org/10.1007/978-3-662-61889-9_9

https://doi.org/10.1007/978-3-662-61889-9_10

https://doi.org/10.1007/978-3-662-61889-9_11

https://doi.org/10.1007/978-3-662-61889-9_12

(C) Springer-Verlag GmbH Deutschland, ein Teil von Springer

Nature 2021

B. Just, Quantencomputing kompakt, IT kompakt, https://doi.org/10.1007/978-3-662-61889-9_13 
Auf S. IX, 2. Absatz von unten: „einfach“ wurde korrigiert zu „einfache“"

Auf S. IX, 2. Absatz von unten: „einen“ wurde korrigiert zu „eine“

Auf S. IX, 1. Fußnote: „beschreiben“ wurde korrigiert zu „beschrieben"

Auf S. X: „Helmut Roth danke ich für das Probelesen und seine sehr“ wurde korrigiert zu „Helmut Roth und Günter Schilling danke ich für das Probelesen und ihre sehr“"

\section{Kapitel 1:}

Auf S. 1, 1. Absatz von oben: Das Komma (,) neben dem Wort „durch“ wurde entfernt.

\section{Kapitel 2:}

Auf S. 10, 1. Absatz: „etwas“ wurde korrigiert zu „etwa“.

Auf S. 10, 1. Absatz: ,und ihren Experimentalfehlerbereinigten Ergebnissen." wurde korrigiert zu "und mit ihren experimentalfehlerbereinigten Ergebnissen."

Auf S. 14: ,u. A.“ wurde korrigiert zu ,u. a.“

Auf S. 15, 3. Fußnote: Das Fragezeichen(?) vor dem Zahl „1“ wurde entfernt.

Auf S. 16, 3. Absatz unter Abschnitt 2.3: ,im folgenden“ wurde korrigiert zu ,in diesem“

Auf S. 16, 4. Absatz unter Abschnitt 2.3: Das Komma (,) neben dem Wort „eingegangen“ wurde entfernt.

Auf S. 17: „Abb. 2.11 zeigt die Situation“ wurde vor der Abbildung 2.11 hochgezogen.

Auf S. 17: „überraschendes“ wurde korrigiert zu „Überraschendes“"

\section{Kapitel 3:}

Auf S. 21, 2. Absatz von oben: Das Komma (,) neben dem Wort „dann“wurde entfernt.

Auf S. 21, 2. Absatz von oben: ,passiert“" wurde neben dem Wort „Photonen“ entfernt. 
Auf S. 23, 1. Absatz unter Abschnitt 3.2: „passieren“ wurde korrigiert zu ,passiert“

Auf S. 23, 1. Absatz unter Abschnitt 3.2: „werden“ wurde korrigiert zu ,wird“

\section{Kapitel 4:}

Auf S. 27, 1. Absatz: „,ihren Kasten“ wurde korrigiert zu „dem Kasten“"

Auf S. 28, 3. Absatz von oben: „liegt“ wurde korrigiert zu ,ist“

\section{Kapitel 5:}

Auf S. 33, 1. Absatz unter Abschnitt 5.2: „passieren“ wurde korrigiert zu ,passiert“"

Auf S. 38, 1. Absatz von oben: „nach“ wurde korrigiert zu ,ist nach "

Auf S. 38, 2. Absatz von oben: „nach“ wurde korrigiert zu ,ist nach “

\section{Kapitel 8:}

Auf S. 54, 3. Absatz von oben: , $\cos 30^{\circ}+\sin 30^{\circ}=1^{\prime \prime}$ wurde korrigiert $\mathrm{zu},,\left(\cos 30^{\circ}\right)^{2}+\left(\sin 30^{\circ}\right)^{2}=1^{\prime \prime}$

Auf S. 54, 5. Absatz von oben: „,sindnatürlich“ wurde korrigiert $\mathrm{zu}$,sind natürlich“

Auf S. 57, 1. Absatz: „ist“ wurde neben dem Wort „Raum“ entfernt.

Auf S. 57, 3. Absatz von oben: ,also“ wurde neben dem Wort „Literatur" entfernt.

Auf S. 59, 1. Absatz: „erst“" wurde korrigiert zu „das erste“

Auf S. 67, 2. Absatz von oben: „Münze 0 war“" wurde korrigiert zu „Münze 1 war““

Auf S. 68, 2. Absatz von oben: ,wiederfinden“ wurde neben dem Wort „Welten“ entfernt.

Auf S. 69, 3. Absatz von oben: ,wie beim“ wurde korrigiert zu „wie es beim“

Auf S. 71, vor Abb. 8.14: Die Formel wurde korrigiert.

Auf S. 72, 9. Zeile von oben: Die Formel wurde korrigiert. 


\section{Kapitel 9:}

Auf S. 77, 2. Absatz von oben: „,beidenQBits“ wurde korrigiert zu „beiden QBits“

Auf S. 80, 2. Absatz: „ditte“ wurde korrigiert zu „dritte“

Auf S. 83, letzter Absatz: „einem kleinen grünen Quadrat“ wurde korrigiert zu ,einem grünen Quadrat“ und ,einem roten kleinen Quadrat“" wurde korrigiert zu ,einem roten Quadrat“"

\section{Kapitel 10:}

Auf S. 90, 1. Absatz: „er“ wurde vor dem Wort „vorher“ entfernt.

\section{Kapitel 11:}

Auf S. 96, 1. Absatz von oben: Anführungszeichen wurden bei dem Wort „spukhafte Fernwirkung“ gesetzt.

Auf S. 102: „u. A.“"wurde korrigiert zu ,u. a.“

\section{Kapitel 12:}

Auf S. 105, 1. Absatz unter Abschnitt 12.1: „Gesetze“ wurde korrigiert zu „Gesetzen“

Auf S. 105, 2. Absatz unter Abschnitt 12.1: „Geforscht wurde“ wurde korrigiert zu „Geforscht wird“

Auf S. 107, 3. Absatz von oben: „die einer“ wurde korrigiert zu "die in einer"

Auf S. 107, 5. Absatz von oben: ,ja“ wurde korrigiert zu ,je“

Auf S. 107, letzten Zeile: „ganzen“ wurde korrigiert zu „Ganzen“

Auf S. 108, 2. Absatz von unten: „,bei auch“ wurde korrigiert zu ,auch bei“"

Auf S. 109, 1. Absatz von oben: „sich“ wurde neben dem Wort „Vorkenntnisse“ entfernt.

Auf S. 109, letzter Absatz unter Abschnitt 12.2: „google bristlecone“ wurde korrigiert zu „Google Bristlecone“

Auf S. 109, letzter Absatz unter Abschnitt 12.2: „google sycamore“" wurde korrigiert zu „Google Sycamore“ 
Auf S. 109, letzter Absatz unter Abschnitt 12.2: „intel Tangle lake“ wurde korrigiert zu „Intel Tangle Lake“

Auf S. 109, 5. Fußnote: „Konzept verschränkte Quanten“ wurde korrigiert zu „Konzept verschränkter Quanten“

Auf S. 111, 1. letzter Absatz: Anführungszeichen wurden bei dem Wort ,spukhafte Fernwirkung“ gesetzt. 